\title{
Radio Recombination Line studies on M82 from LOFAR HBA observations
}

\author{
M. C. Toribio ${ }^{1}$, L. K. Morabito ${ }^{2}$, J. B. R. Oonk ${ }^{1,2}$, F. Salgado ${ }^{2}$, \\ A. G. G. M. Tielens ${ }^{2}$ and H. J. A. Röttgering ${ }^{2}$ \\ ${ }^{1}$ Netherlands Institute for Radio Astronomy (ASTRON), \\ Postbus 2, 7990 AA Dwingeloo, The Netherlands \\ email: toribio@astron.nl \\ ${ }^{2}$ Leiden Observatory, Leiden University, \\ P. O. Box 9513, 2300 RA Leiden, The Netherlands
}

\begin{abstract}
We continue our search for extragalactic Carbon Radio Recombination Lines (CRRL) in M82 with the LOw Frequency ARray (LOFAR; van Haarlem et al. 2013). The goal of our project is to determine the physical conditions of the cold neutral gas in this object, for which low frequency radio recombination lines can provide a sensitive probe.
\end{abstract}

Keywords. galaxies: ISM

LOFAR is capable of detecting CRRLs in extragalactic sources. The first ever extragalactic detection of these lines was achieved by us using data taken with LOFAR's Low Band Antenna (LBA) in the 48-64 MHz frequency range in the nearby starbursting galaxy M82 (Morabito et al. 2014). By studying the frequency behaviour of these CRRLs at low frequencies $(<1 \mathrm{GHz})$ we can constrain the physical properties (temperature, density and abundances) of the cold, neutral medium (e.g., Walmsley \& Watson 1982).

We are now expanding our search for CRRLs to the frequency range 110-190 MHz with the LOFAR High Band Antennae (HBA). First tests indicate that we reach a spectral $\mathrm{rms} \sim 10^{-3}$ per $6 \mathrm{~km} / \mathrm{s}$. By stacking $\sim 20$ lines we should be able to detect CRRLs from M82 at these frequencies. Futhermore, the unprecedented sensitiviy and resolution of the HBAs ( $\sim 5$ arcsec) will allow us to identify the location of the CRRL emitting gas.

\section{Acknowledgements}

MCT acknowledges financial support from LKBF. LKM acknowledges financial support from NWO Top LOFAR project, project n. 614.001.006. JBRO acknowledges financial support from NWO Top LOFAR-CRRL project, project n. 614.001.351.

\section{References}

van Haarlem, M. P., Wise, M. W., Gunst, A. W., et al. 2013, A\&EA, 556, A2

Morabito, L. K., Oonk, J. B. R., Salgado, F., Toribio, M. C., Tielens, A. G. G. M, \& and Röttgering, H. 2014, Proc. of the IAU Symposium No. 309: Galaxies in 3D across the Universe, B. L. Ziegler, F. Combes, H. Dannerbauer, M. Verdugo, eds. (Cambridge University Press)

Walmsley, C. M. \& Watson, W. D. 1982, ApJ, 260, 317 